\title{
Note
}

\section{Dissociations in rod bisection: The effect of viewing conditions on perception and action}

\author{
Laura E. Hughes ${ }^{a, *}$, Timothy C. Bates ${ }^{a}$ and Anne M. Aimola Davies ${ }^{a, b}$ \\ ${ }^{a}$ Macquarie Centre for Cognitive Science, Macquarie University, Sydney, Australia \\ ${ }^{\mathrm{b}}$ School of Psychology, The Australian National University, Canberra, ACT 0200, Australia
}

\section{A R T I C L E I N F O}

Article history:

Received 26 May 2005

Reviewed 10 August 2005

Revised 14 March 2006

Accepted 15 March 2006

Action editor Yves Rosetti

Published online 30 January 2008

Keywords:

Visual illusion

Pseudoneglect

Binocular

Monocular

Visual occlusion

\begin{abstract}
A B S T R A C T
Research providing evidence from patients and neurologically healthy participants has demonstrated that visual perception can dissociate from visually guided actions, and that this dissociation can be removed by reducing visual feedback to a monocular view, or by completely occluding vision. Previously we have demonstrated a similar dissociation between perception and action on a rod-bisection task. The current paper examines whether manipulating the viewing conditions can also affect this dissociation. Forty-eight right-handed participants bisected five rods of different lengths, by pointing to the centre and by picking each up by the centre, under three viewing conditions: binocular viewing, monocular viewing and occluded viewing. Binocular viewing resulted in the expected perception-action dissociation: pointing bisection errors were to the right of centre and grasping bisection errors showed no bias. However, this pattern was also evident for the monocular-viewing condition, demonstrating that monocular viewing had no significant effect on bisection. In contrast, complete visual occlusion led to the elimination of the perception-action dissociation, and, in addition, the direction of the pointing errors reversed: both pointing and grasping errors were to the left of centre. These results are compared with a line-bisection task performed under similar conditions. This task resulted in consistent biases for which reducing visual feedback only influenced the extent of the error. The direction of the error was influenced by line position. These results demonstrate that theories for differential processing in the ventral and dorsal streams, used to elucidate perception-action dissociations, may not be compatible with the rod-bisection task and that online visuomotor feedback may better explain the dissociation.
\end{abstract}

(c) 2007 Elsevier Srl. All rights reserved.

\section{Introduction}

Bisection tasks have frequently been used as measures of spatial attention. Results vary across studies but a common finding is 'pseudoneglect', a term coined by Bowers and Heilman (1980) to reflect the tendency of neurologically healthy participants to bisect lines slightly to the left of centre. A meta analysis by Jewell and McCourt (2000) revealed that line bisection tends to result in a leftward bias.

Several theories of asymmetrical activation for spatial attention have been used to provide explanations for this leftward shift. The activation-orientation hypothesis (Kinsbourne, 1970) suggests that preferential orientation to one side of space is related to hemispheric specialization for

\footnotetext{
* Corresponding author. MRC-CBU, 15 Chaucer Road, Cambridge CB2 7EF, UK.

E-mail address: laura.hughes@mrc-cbu.cam.ac.uk (L.E. Hughes). 0010-9452/\$ - see front matter ( 2007 Elsevier Srl. All rights reserved. doi:10.1016/j.cortex.2006.03.003
} 
different tasks and that activation or arousal of one hemisphere biases attention to the contralateral side. The right hemisphere is thought to be dominant for attention (Heilman and Van den Abel, 1980), and although both the right and left hemispheres can orient attention to the contralateral hemispace, a slight leftward predominance can occur due to greater neuronal resources in the right hemisphere for spatial attention (Mesulam, 1999). Line bisection is thought to require spatial attention, resulting in the leftward bias (Bowers and Heilman, 1980; Reuter-Lorenz et al., 1990).

In contrast with these bisection studies, we (Hughes et al., $2004,2005)$ recently reported that neurologically healthy participants tend to bisect rods to the right when making perceptual judgements about the centre. Earlier work by Hjaltason et al. (1993) also reported rightward errors in rod bisection for patients with neglect but also small rightward errors for the control group. This is a pattern unsurprising for neglect patients but unusual for control participants. In addition to this rightward bias in rod bisection, we demonstrated that bisections made when participants picked the rods up by the centre were not biased. Similar dissociations in spatial attention and spatial actions have also been reported for both neurologically healthy participants and patients with visuospatial neglect. Ellis et al. (1999) used the Judd illusion to show that, while perceptual judgements of the centre of the shaft (a bar) were shifted in the direction of the illusion, lifting the bar by the centre resulted in a more accurate hand position.

This dissociation on the rod-bisection task has also been demonstrated in patients with visuospatial neglect. Patients revealed rightward errors when pointing to the centre of a rod but showed a reduction in errors when they were asked to lift the rods by the centre (Robertson et al., 1995; Edwards and Humphreys, 1999). Other tasks examining patient behaviour have revealed further dissociations between perception and action. McIntosh et al. (2004) showed that patients with neglect were impaired at pointing midway between two objects but were not impaired when reaching between the two objects. The opposite pattern of performance has been shown for patients with optic ataxia, who were able to accurately bisect the space between two objects yet had difficulty reaching between the objects (Schindler et al., 2004; Rossetti et al., 2005).

One theory considered by the above studies to explain these patterns of behaviour is the two stream theory of Milner and Goodale (1995). They propose that the ventral and dorsal streams utilize perceptual information differentially for perceptual judgements and visually guided actions. The ventral stream is thought to provide the representations used for perceptual judgements: these representations are proposed to exemplify the stable visual characteristics of objects and are used to provide long lasting knowledge of the object and its spatial location and relationship to the environment. In contrast, the dorsal stream is suggested to be responsible for visually guided actions: these representations are short lived, not lasting longer than a couple of seconds, and are refreshed constantly by visual feedback. This view is tested by the plethora of studies examining both patients and neurologically healthy populations. Many studies have used visual illusions to tease apart ventral and dorsal processes, demonstrating a distinct dissociation between perceptual judgements which are influenced by illusions and visually guided actions which are not. However, only some results are used to support the two stream theory (see Smeets et al., 2002; Franz et al., 2000; Bruno, 2001; Carey, 2001).

In patients with visuospatial neglect the dissociations are thought to arise because actions are based on dorsal representations in superior parietal areas, rather than on areas with lesions such as inferior parietal or other neuroanatomical areas causing neglect (Milner and Goodale, 1995; Robertson, 1999). Although patient data can support this assertion, the sparing does not apply to all (McIntosh et al., 2004). Interestingly, the studies on patients tend to use pointing responses to represent perceptual judgements, whereas the studies on illusions with neurologically healthy participants have used pointing responses to represent visually guided actions. It has been argued that manual estimates, involving hand and finger movements to portray perception, do not recruit the visuomotor network but just provide a "manual readout of what [participants] perceive" (Haffenden and Goodale, 1998, p. 125). Thus pointing may fall into either category depending on the task.

An alternative explanation for the dissociation on rod bisection is discussed by Edwards and Humphreys (1999), who found that their patient with neglect, MP, showed an improvement in bisection when grasping only under full visual guidance. When vision of his hand and target were removed, both pointing and grasping responses were similarly biased to the right of the rod. The authors suggest that the improvement in grasping under full vision could be due to online visuomotor adjustment, which results in the consequent improved performance.

Studies with neurologically healthy participants have also revealed that removing visual feedback affects the accuracy of grasping (Westwood and Goodale, 2003; Hu and Goodale, 2000; Westwood et al., 2000a), although these studies generally support the two stream theory. It is argued that grasping, after vision of the object is removed, relies on ventral representations because only these representations are stored for a significant length of time. A number of studies have reported that even a short delay between viewing the stimulus and the onset of action can affect accuracy (Bridgeman et al., 1997, 2000; Westwood et al., 2000b, 2001; Westwood and Goodale, 2003), whereas, whenever actions were initiated prior to the visual occlusion, there was no effect (Hu and Goodale, 2000).

The type of visual feedback may also be an influencing factor on the accuracy of actions. Binocular input is suggested to be important for visually guided actions. Performing actions under monocular viewing has been shown to increase movement duration, reduce peak velocity (which is also achieved earlier than with binocular viewing), and increase time spent decelerating (Loftus et al., 2004; Jackson et al., 2002; Servos et al., 1992; Servos and Goodale, 1994). In addition, grip aperture may be wider or narrower under monocular viewing (Jackson et al., 1997; Mon-Williams and Dijkerman, 1999), and this effect on aperture may depend on whether the target is constantly in view or appears suddenly (Loftus et al., 2004). The effect of monocular vision on actions may be due to the greater reliance on pictorial cues to calculate the distance of an object, rather than vergence cues available in binocular vision (Marotta et al., 1998). 
Thus objects can still be manipulated under monocular vision but a different method for calibrating size and distance may be used.

This difference has been highlighted in studies examining patients with visual agnosia and neurologically healthy participants. Monocular viewing can significantly affect patients' ability to judge the distance of a target (Mon-Williams et al., 2001) and can considerably disrupt constancy of grip aperture (Marotta et al., 1997). Such difficulties are thought to arise because the analysis of pictorial cues is a ventral stream task and patients with visual agnosia have damage to their ventral stream and consequently the representations are disrupted. Marotta et al. (1998) report an effect of monocular vision on neurologically healthy participants. When grasping the central disc of an Ebbinghaus array, participants were only able to scale their grasp appropriately in binocular-viewing conditions. Under monocular viewing, grasp was influenced by the illusion. Again, this is suggested to occur because monocular viewing results in a greater reliance on pictorial cues provided by the ventral stream. Under binocular vision, grasp is not influenced by illusions because accurate size and distance can be calibrated by the dorsal stream using cues such as vergence.

The aim of the current experiment was to examine whether the dissociations between perception and action that have been observed in the rod-bisection task could be affected by reducing or removing visual feedback, and whether this would have any bearing on the cause of the dissociation. Previous studies have shown that under reduced vision participants are still able to complete tasks, but show a change in their pattern of behaviour. Hence, in the current study we also expect participants to be able to complete rod bisection under reduced vision, but expect a shift in the pattern of bisections due to a greater reliance on a different method for processing the stimuli. One prediction is that the dissociation on the rod-bisection task is due to the use of different visual streams to complete each task. Another prediction (Edwards and Humphreys, 1999) is that the dissociation could be due to the use of online visuomotor feedback for grasping. If the dissociation on the rod-bisection task is due to the use of different ventral and dorsal representations, then, under both monocular- and occluded-viewing conditions, grasping bisections should rely more on ventral representations and the pattern of bisections should fall inline with that of the pointing task. If the dissociation is due to online visual feedback, then monocular viewing should have no or little effect on grasping, but occluded viewing should result in pointing and grasping errors in the same direction. In both cases pointing errors are expected to stay constant because first, these bisections should be based on ventral representations regardless of viewing condition and second, they are not thought to be affected by visuomotor feedback.

\section{Materials and methods}

\subsection{Design}

A repeated measures design was used to compare bisection of five rods (of different lengths) by pointing to the centre and by grasping the centre of each under three different viewing conditions: binocular, monocular and occluded. Three sets of 10 trials were completed by each participant. The pointing bisection task involved the participant pointing to the midpoint of each rod, and the grasping bisection task involved the participant grasping or lifting the rod by the centre. In the binocular-viewing condition, all bisections were made with both eyes open. In the monocular-viewing condition, all bisections were made with only the dominant eye open. The head position was either free, to allow free viewing, or fixed, to prevent head movements compensating for loss of binocular vision. In the occluded-viewing condition, both eyes were closed after the rod had been viewed, and the bisection made after a $5 \mathrm{sec}$ delay. The measures taken were calculated as millimeters from true centre. The presentation of the different rods was randomized. For each viewing condition, participants either completed the pointing tasks first or the grasping tasks first. The viewing conditions were presented in a specific order, binocular, monocular and then occluded viewing. It was expected that the monocular- and occludedviewing conditions would be much harder, and the experience of completing the tasks under binocular viewing first may minimize the error variance.

In addition to the rod-bisection tasks, two assessments of line bisection were used. The first, completed by all participants, assessed the 'normality' of visuospatial perception using a paper-and-pencil line-bisection task, for which there is much normative data provided in the literature. The second, completed by 16 participants, examined computerized line bisection under the three viewing conditions. The lines were presented on a touch-screen monitor and participants used a small pen-like pointer to indicate the centre of each line.

\subsection{Participants}

Forty-eight 1st year undergraduate students, 25 females (mean age 20; age range 18-27) and 23 males (mean age 20.4; age range 18-28) participated in the rod-bisection tasks. Of these participants, 16 (eight females and eight males) completed the additional computerized line-bisection task. All provided informed consent and in return for course credit. The study was approved by the Human Research Ethics Committee and tests were conducted in accordance with the ethical standards laid down in the 1964 Declaration of Helsinki. All participants were right-handed, as assessed by self-report from a seven-item questionnaire adapted from the Edinburgh Handedness Inventory (Oldfield, 1971). Extreme right-handedness was indicated by a maximum score of +100 , the mean laterality score of the participants was +92.9 , with a range of +57.2 to +100 . Measures of eye dominance were also taken using a 'tube test'. Participants were asked to look through a narrow cardboard tube with both eyes open and focus on a target positioned straight ahead. When the target was in focus the participants were instructed to close one eye. If the target remained in focus, the open eye was recorded as the dominant eye. Thirty-two participants were right-eye dominant and 16 left-eye dominant. 


\subsection{Apparatus and material}

The rod-bisection tasks used five black wooden rods $(2 \mathrm{~cm}$ in circumference) of five lengths $(25-45 \mathrm{~cm}$ in $5 \mathrm{~cm}$ increments). Each rod weighed between $35 \mathrm{~g}$ and $40 \mathrm{~g}$. One side was covered with black Velcro and on another side a ruler was printed to facilitate accurate recording. All responses were made using a small pointer, that is, a ring made of Velcro worn on the index finger. On contact with the rod, the ring stuck to the Velcro and a small arrow affixed to the ring indicated the central point. For the monocular-viewing condition a headrest was used to prevent head movements.

The paper-and-pencil line-bisection task was presented on sheets of A4 paper in landscape orientation. Six lines $(10 \mathrm{~cm}$ in length) were presented individually to the centre, to the right and to the left of centre (for the latter two cases the midpoint of each line was shifted from centre by $\pm 8 \mathrm{~cm}$ ). The computerized line-bisection task was presented on a touch-screen monitor. Lines $(10 \mathrm{~cm})$ were presented six times in each of five positions: far left, left, centre, right and far right. In the off-centre positions, the centre of the line was offset by $\pm 5 \mathrm{~cm}$ and $\pm 10 \mathrm{~cm}$. The computer line-bisection task was also presented three times, once in the binocular condition, once in the monocular condition (for which the headrest was again used to prevent head movements compensating for loss of binocular vision) and once in the occluded condition.

\subsection{Procedure}

All participants completed each task using their right, and dominant, hand. The paper-and-pencil line-bisection task was presented first. Participants sat at a $1 \mathrm{~m}$-by- $2 \mathrm{~m}$ table opposite the experimenter with the sheets of paper presented directly in front of them. They were asked to draw a short vertical line through the centre of each line on the page.

Sixteen participants (of the 48) then completed the computerized line-bisection task. The lines were presented first in the binocular-viewing condition and participants were asked to use the pointer to indicate the centre of each line. The test was then rerun with participants instructed to close their non-dominant eye while making the bisections. All participants wore an eye patch to ensure occlusion of the non-dominant eye. To control for head movements, which may compensate for the loss of binocular vision, participants were requested to place their head in a headrest which minimized movement. In the final condition, participants completed the bisections under the occluded-viewing condition. They were instructed to look at the line for approximately $5 \mathrm{sec}$, remember the location of the centre, then close their eyes for a count of five (ensuring that movements were based on memory), and with their eyes closed point to the centre of the line. In the case where participants' decisions were more than $\pm 5 \mathrm{~cm}$ from the actual line, no response was recorded and the bisection was attempted again until within the specified range. Interestingly, participants were surprisingly accurate with their eyes closed. All measurements taken were the errors from centre.

All participants then completed the rod-bisection tasks. Rods were placed directly in front of the participant, approximately at their midsagittal plane and $20 \mathrm{~cm}$ from the edge of the table. In the binocular-viewing condition, the pointing task required participants to use their right forefinger (on which the pointer was worn) to point to the centre of the rod and the grasping task required the participants to make one smooth natural grasping movement - using a pincer like grip with their right forefinger and thumb - to pick the rod up by the centre. Measurements of the position of the pointer were taken immediately after each bisection. In all tasks, participants had viewed the rod for approximately $5 \mathrm{sec}$ before making a decision in all conditions.

In the monocular-viewing condition, the pointing and grasping bisections were completed as above but with the participant closing their non-dominant eye while viewing each rod and performing the bisections. For this condition, participants were split into two groups. One group of 16 participants completed the task with their head position fixed (using a headrest), to control for head movements which may compensate for loss of binocular vision. The remaining participants completed the task, as in the previous conditions, under free viewing.

In the occluded-viewing condition, participants were presented with a rod under full view, they viewed the rod for approximately $5 \mathrm{sec}$ then closed both eyes. After closing both eyes a 5-sec delay was initiated before the participant could point to or grasp the centre of each rod. Participants looked at their decision before it was recorded so that they received visual feedback as they had in the other two viewing conditions. This procedure was repeated with each of the five rods. In both the monocular- and occluded-viewing conditions, the participants were carefully watched by the experimenter to ensure they conformed to the instructions. No participants had to be removed or be retested for noncompliance.

\section{Results}

\subsection{Line-bisection task}

For the paper line-bisection task, errors for lines positioned on the left side of the page deviated significantly to the left (mean error $\left.=-2.04 \mathrm{~mm}, \mathrm{SE}=.58, \mathrm{t}_{(47)}=-3.5, p<.01\right)$. The central line and the rightward lines showed no significant bias (central line: mean error $=-.6 \mathrm{~mm}, \mathrm{SE}=.4, \mathrm{t}_{(47)}=-1.3, p>.05$ and right line: mean error $\left.=.5 \mathrm{~mm}, \mathrm{SE}=.6, \mathrm{t}_{(47)}=.98, p>.05\right)$.

For the computer line-bisection task, a repeated measures analysis of variance (ANOVA) was performed to examine differences between the three viewing conditions (binocular, monocular and occluded) and the five positions of the lines (far left, left, centre, right, and far right). There was no effect of viewing condition $\left(F_{(2,30)}=.57, p>.05\right)$ but line position was significant $\left(F_{(4,60)}=19.4, p<.01\right)$ and there was a significant interaction between viewing condition and position $\left(F_{(8,120)}=10.1, p<.01\right)$. Fig. 1 shows the line-bisection data for each line position across the three viewing conditions. The interaction between viewing condition and line position is reflected in the much greater errors when the lines are positioned off centre, and when vision is occluded. Leftward 


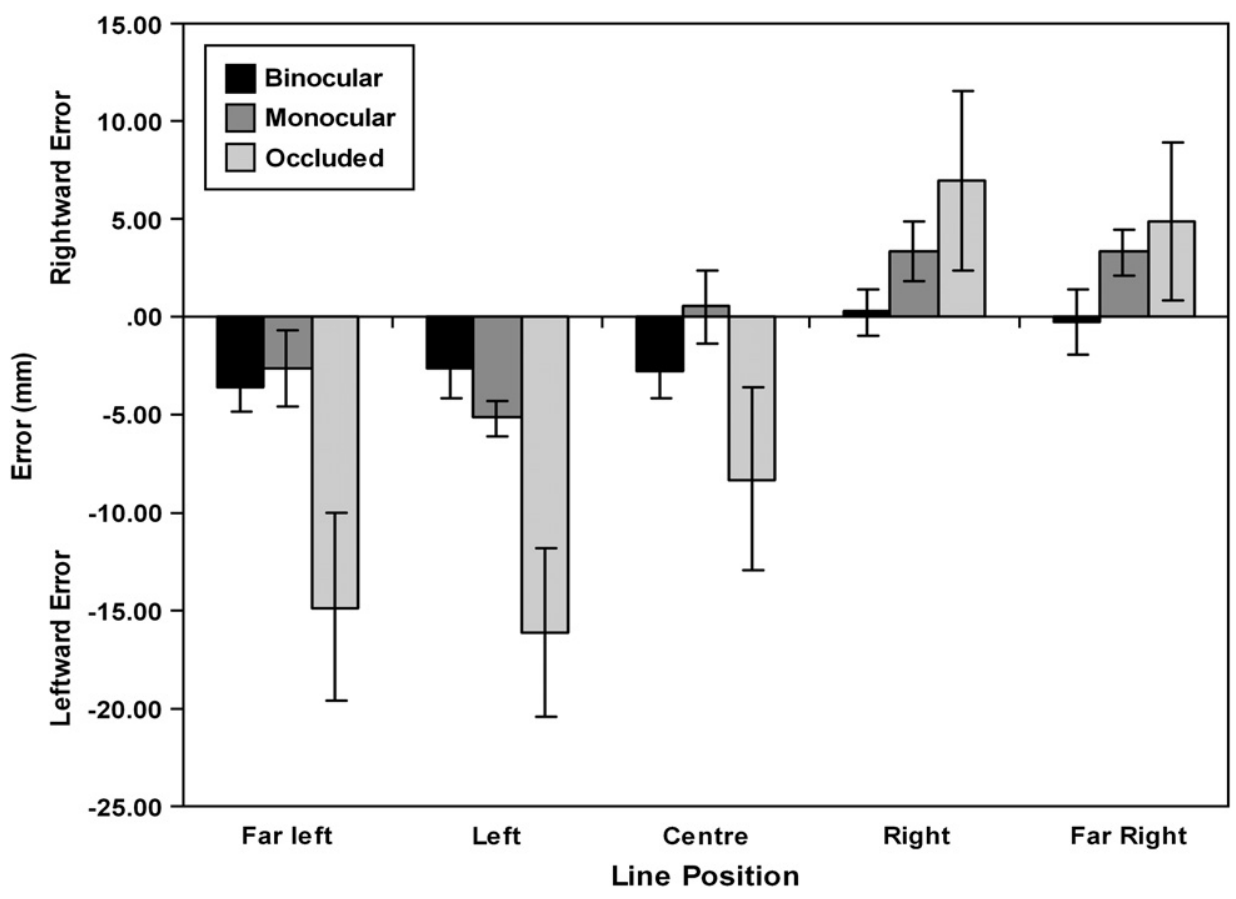

Fig. 1 - Line-bisection errors in millimeters for each of the five positions across the three viewing conditions with standard error bars. The lines positioned on the far left and left side of the screen result in leftward errors in all conditions, the lines in the centre also show a general leftward trend in the errors (for binocular and occluded conditions) and those on the right and far right result in rightward errors (for the monocular and occluded conditions).

errors in the occluded condition particularly appear to be exaggerated when the rod is positioned on the left.

When the data were collapsed across viewing condition, significant directional errors were revealed. The lines positioned on the left resulted in leftward errors (far left: mean $=-7 \mathrm{~mm}, \quad \mathrm{SE}=2.0, \quad \mathrm{t}_{(15)}=-3.5, \quad p<.01$ and left: mean $\left.=-7.9 \mathrm{~mm}, \mathrm{SE}=1.3, \mathrm{t}_{(15)}=-5.8, p<.01\right)$. The lines in the centre revealed no significant bias (mean $=-.9 \mathrm{~mm}$, $\left.\mathrm{SE}=1.6, \mathrm{t}_{(15)}=-.6, p>.05\right)$. The lines on the right revealed a rightward trend, which was only significant in the 'right', and not the 'far right' position (right: mean $=3.4 \mathrm{~mm}$, $\mathrm{SE}=1.5, \mathrm{t}_{(15)}=2.2, \quad p<.05$ and far right: mean $=2.3 \mathrm{~mm}$, $\left.\mathrm{SE}=1.3, \mathrm{t}_{(15)}=1.7, p>.05\right)$. Posthoc analysis also revealed that the extent of the bias was greater for lines positioned on the left than for lines positioned on the right for all three viewing conditions (binocular: $t_{(15)}=2.7, p<.05$; monocular: $t_{(15)}=3.9, p<.01$ and occluded $\left.t_{(15)}=5.2, p<.01\right)$.

\subsection{Rod-bisection task}

We first examined the monocular-viewing conditions for an effect of fixing the head position compared to free viewing. There was no effect of these two conditions for either pointing (free viewing: mean $=.6 \mathrm{~mm}, \mathrm{SE}=.6$ and fixed viewing: mean $=1.4 \mathrm{~mm}, \mathrm{SE}=.9 ; \mathrm{t}_{(46)}=-.6, p>.05$ ) or grasping (free viewing: $\operatorname{mean}=-1.4 \mathrm{~mm}, \mathrm{SE}=.7$ and fixed viewing: mean $\left.=-.4 \mathrm{~mm}, \mathrm{SE}=1.0 ; \mathrm{t}_{(46)}=-.7, p>.05\right)$. Thus the data were collapsed across this measure for the following analyses.

A repeated measures three-factor ANOVA was used to analyze task (pointing or grasping) by viewing condition (binocular, monocular or occluded) by rod length (five lengths of $25-45 \mathrm{~cm}$ ). Viewing eye was examined as a covariate. There was a significant effect of task $\left(F_{(1,46)}=7.6, p<.01\right)$ but no significant effect of viewing condition $\left(F_{(2,92)}=2.2, p>.05\right)$, and no effect of rod length $\left(F_{(4,184)}=1.2, p>.05\right)$. There was a significant interaction between task and viewing condition $\left(F_{(2,92)}=3.4, p<.05\right)$. Fig. 2 shows where the main differences lie across the task and the viewing conditions. While there was no effect of eye dominance on viewing condition, there was a significant effect of eye dominance on task $\left(F_{(1,46)}=5.0\right.$, $p<.05)$, and an interaction between eye dominance, task and viewing condition $\left(F_{(2,92)}=5.8, p<.05\right)$. There were no other significant interactions.

The significant effects were examined in further analyses of the data. As there were no significant differences in the extent of the errors between the five-rod lengths, the data were collapsed across this measure.

A repeated measures ANOVA was used to examine the interaction between task and viewing condition. For the pointing task, there was a significant difference for the three conditions: binocular: mean $=1.6 \mathrm{~mm}, \mathrm{SE}=.6$; monocular: mean $=.9 \mathrm{~mm}, \mathrm{SE}=.6$ and occluded: mean $=-2.7 \mathrm{~mm}$, $\mathrm{SE}=1.4 ; F_{(2,94)}=8.1, p<.01$. A similar ANOVA was also used to examine how the task of grasping changes across the three viewing conditions, but no significant differences were revealed (binocular: mean $=-.5 \mathrm{~mm}, \mathrm{SE}=.7$; monocular: mean $=-1.1 \mathrm{~mm}, \mathrm{SE}=.6$ and occluded: mean $=-2.3 \mathrm{~mm}$, $\left.\mathrm{SE}=1.2 ; F_{(2,94)}=1.3, p>.05\right)$.

In the binocular-viewing condition, the pointing errors were significantly different from zero, revealing a rightward 


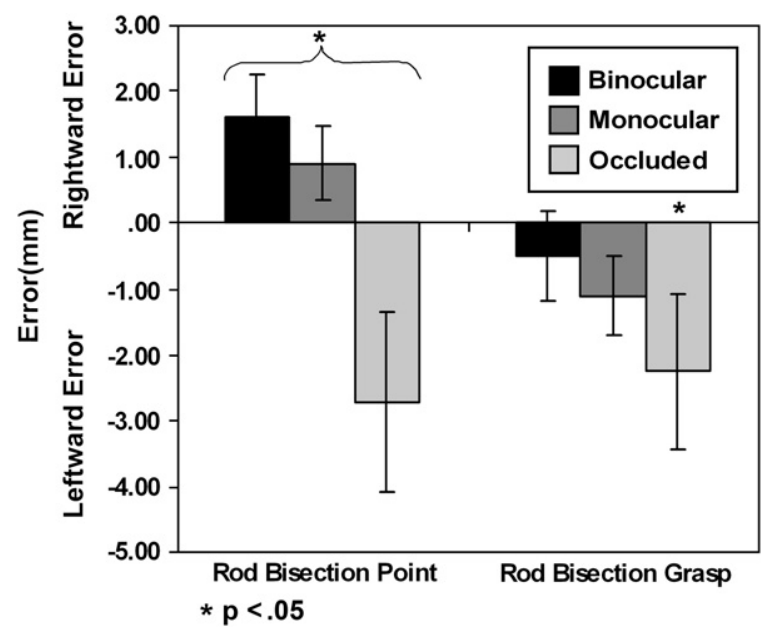

Fig. 2 - The mean bisection errors in millimeters with standard error bars. The results are collapsed over rod length, which did not have any effect on bisection error. In the rod-bisection pointing task, a rightward trend occurs for both the binocular- and monocular-viewing conditions but a leftward bias is evident for the occluded-viewing condition and for all the grasping conditions.

bias $\left(t_{(47)}=2.5, p<.05\right)$. These pointing errors were significantly different from the grasping errors $\left(t_{(47)}=3.7, p<.01\right)$, which showed no significant bias in either a leftward or rightward direction. In the monocular-viewing condition, the errors in pointing and grasping were also significantly different from each other $\left(t_{(47)}=3.7, p<.01\right)$ but again not significantly different from zero, thus indicating no directional bias. In the occluded-viewing condition, the two tasks were not significantly different from each other $\left(t_{(31)}=-.3, p>.05\right)$ and the leftward trend reached significance (pointing: $\mathrm{t}_{(47)}=-2.0, p=.05$ and grasping: $\left.\mathrm{t}_{(47)}=-1.9, p=.05\right)$.

\subsection{Correlations}

The binocular and monocular pointing and grasping conditions were all positively correlated, and the occluded pointing and grasping also positively correlated with each other, but not with the other conditions. See Table 1 for details.

\subsection{Eye dominance}

Thirty-two participants were right-eye dominant and 16 were left-eye dominant, and all performed the monocular-viewing condition using their dominant eye. In the initial ANOVA an interaction between eye dominance and task was revealed. Further examination of this found no significant differences between the two groups on all of the six bisection measures, including the two monocular-viewing conditions. However, when the data were collapsed across viewing conditions, it appears that in the pointing task, left-eye-dominant participants point slightly more to the right (mean $=.2 \mathrm{~mm}$ ) than right-eye-dominant participants (mean $=-2 \mathrm{~mm}$ ) and, in the grasping task, left-eye-dominant participants grasp further to the left (mean $=-2.7 \mathrm{~mm}$ ) than those who are righteye-dominant (mean $=-.8 \mathrm{~mm}$ ).

\section{Discussion}

Participants showed a leftward trend in errors when bisecting lines, which was modulated to a rightward bias when the lines were moved to the right, thus our subject pool appears 'normal' in this respect and consistent with previous reports in the literature (cf. Jewell and McCourt, 2000). These biases were also evident when participants viewed the lines with only their dominant eye, in the monocular condition, and when they performed the bisections with their eyes closed. The bisection errors showed a centrifugal effect (that is, greater leftward errors for lines presented on the left, and greater rightward errors for lines presented on the right). There was also a greater directional bias for lines positioned on the left than on the right. This bias could be explained by an interaction of line position, orientation to the contralateral side of space, as predicted by the activation-orientation hypothesis (Kinsbourne, 1970) and the spatial nature of the task increasing right-hemisphere activation. Interestingly, in the occluded condition, not only were the direction of the errors exacerbated for lines on the left and right, but leftward errors were also increased for lines presented at the centre.

In the rod-bisection task there were significant differences between the pointing and grasping conditions: pointing bisections resulted in a significant rightward bias and grasping

Table 1 - Pearson's $r$ correlations between the two bisection tasks and three viewing conditions

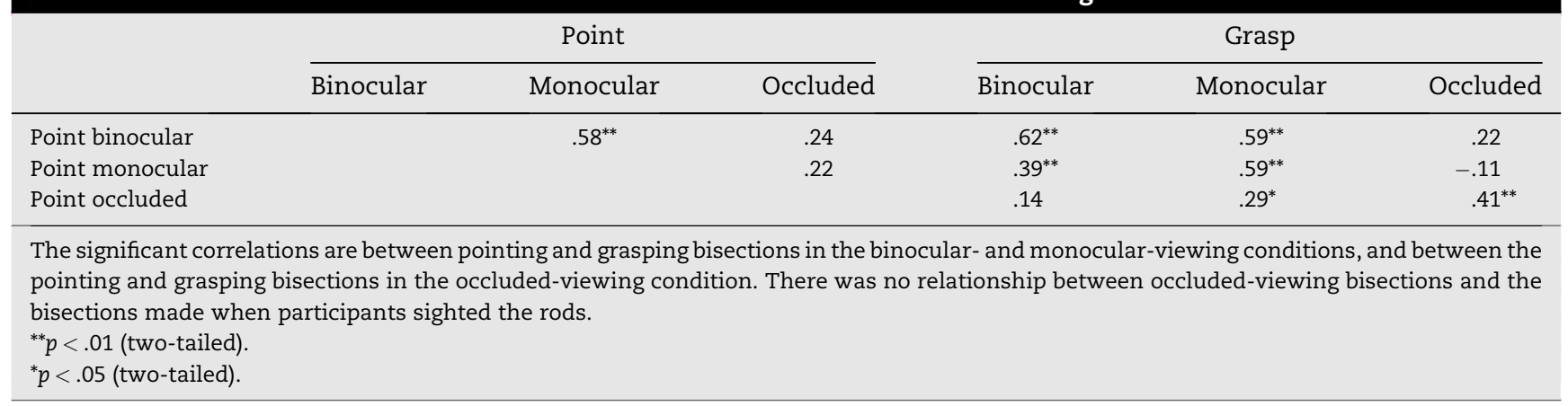


bisections resulted in a non-significant directional bias. These effects of task type (pointing or grasping) on directional bias are similar to previous reports (Hughes et al., 2004, 2005). The rightward bias of pointing is not thought to be a mechanical bias, as left-handed bisections also result in small rightward errors (Hughes, 2005). The effect of eye dominance on bisection may have had a small influence on the direction of bisection as left-eye dominant participants had a greater bias to the right side than right-eye dominant participants. Eye dominance has previously been reported to affect bisection, although past results are not consistent (see Jewell and McCourt, 2000 for a review).

The pattern of bisections under the different viewing conditions was unexpected. Specifically, bisections under binocular and monocular viewing were similar, and monocular viewing did not significantly affect the errors for pointing or grasping. This is reflected in the positive correlations between the binocular and monocular conditions. The finding cannot be attributed to head movements, which could be used to compensate for the lack of binocular vision, since the results for the fixed- and free-viewing conditions did not differ. The most parsimonious explanation simply suggests that monocular viewing does not affect the extent or direction of error for rod bisection. An effect on grasping was predicted because past research indicates that monocular viewing affects grasp dynamics: under a reduced vision, calculating accurate size and distance may rely more on pictorial cues provided by the ventral stream, than cues such as vergence used by the dorsal stream (Marotta et al., 1998). The lack of difference between the binocular and monocular conditions in the rod-bisection task suggests that the dissociation between pointing and grasping is not due to the use of ventral versus dorsal stream in the two tasks. The dissociation may instead result from online visuomotor feedback, for which the occluded condition provides more information on whether this could be driving the dissociation.

When visual feedback of hand and stimuli was occluded, the dissociation between pointing and grasping disappeared, which was as we predicted. We also predicted that occlusion would result in the pattern of bisections changing for the grasping condition, but not for the pointing condition. Studies examining the effect of illusions on perception and action have shown that when vision of the object is removed, visually guided actions show an influence of the illusion which is not present under binocular vision (e.g., $\mathrm{Hu}$ and Goodale, 2000; Westwood et al., 2000a; Westwood and Goodale, 2003). Westwood and Goodale (2003) suggest that this is due to both perception and action relying on the same ventral representations, which are susceptible to the illusions.

Conversely, our results show that removing visual feedback also affected the pointing bisections, and there was no significant change in the pattern of grasping the rod by the centre. The leftward errors for grasping are greater in this condition than in the binocular and monocular conditions, but not significantly. It seems that, under the occluded condition, both pointing and grasping may rely on the same representation and thus the leftward errors in bisection are similar. When visual feedback is available these errors are shifted rightward by a small amount for grasping and by a significant amount for pointing. The effect of visual feedback is also evident in the lack of correlation between the occluded-viewing condition and the monocular- and binocular-viewing conditions, indicating that this is a very different task.

These results appear a better fit with Edwards and Humphreys (1999) theory of visual feedback, than with the two stream theory. Edwards and Humphreys suggest that their patient with neglect, MP, used the same representation at movement outset for pointing and grasping but the final trajectory of the hand was altered by the visual feedback available for grasping the rod, so that the grasp was more accurate. When feedback was not available, MP had to rely on the initial impaired representation, which could not be updated as the action progressed. Similarly, in our study, under visual occlusion, participants may rely on the same (leftward biased) representation for both pointing and grasping. When visual feedback was available, either monocularly or binocularly, both pointing and grasping responses were affected, but during the grasp the feedback could be used to alter the trajectory of the hand so that the final position was not biased to one side.

Visual feedback also appears to have another unexpected effect: the rightward shift in rod bisection provides a direct contrast with the leftward shift in line bisection. The linebisection task produced consistent biases across the three viewing conditions, which is compatible with evidence of a leftward bias in the literature under both visual and nonvisual conditions. Jewell and McCourt's (2000) meta analysis revealed that tactile-bisection tasks and midsagittal pointing tasks lead to leftward errors. Tactile bisection is somewhat comparable to our rod-bisection task under occluded viewing, that is, both tasks are completed with no visual feedback during the movement. When visual feedback is available, the bisections are shifted in the opposite direction but to different extents for pointing and grasping.

We also considered whether the leftward error may be related to the effects of midsagittal pointing tasks. Birch et al. (1960) reported that when participants were asked to point straight ahead, under occluded vision they pointed to their left but with full vision they pointed to their right. However, the findings in the literature are not consistent. Jeannerod and Biguer (1987) found that under full vision participants pointed to their left in a midsagittal pointing task. It is unlikely that participants in our study were pointing to their midsagittal plane rather than the centre of the line or rod, if they were, we would expect a similar extent of errors for the lines and rods. Instead, line-bisection errors were on average actually much larger than rod-bisection errors. Also, participants rarely had difficulty placing their finger on the object.

In conclusion, the results do indicate that the two stream theory cannot by itself be utilised to explain the dissociation on the rod-bisection task. If both pointing and grasping responses rely on the same representation, then the differences may be due to the way in which visual feedback is used. Why this should be more evident for pointing and not for grasping needs to be explored further. Another factor to be examined in more detail concerns the rightward bias for rod bisection which contrasts with the leftward bias for line bisection. Although the current study does not address the cause of the rightward bias in rod bisection, several explanations are presented in earlier papers (Hughes et al., 2004, 2005). 


\section{Acknowledgements}

This work was supported by an iMURS scholarship from Macquarie University, Sydney. The authors wish to thank the students who participated in the experiments and $\mathrm{Mel}$ Goodale and an anonymous reviewer for their invaluable comments on the design.

\section{R E F E R E N C E S}

Birch HG, Proctor F, Bortner M, and Lowenthal M. Perception in hemiplegia II: judgement of the median plane. Archives of Physical Medicine and Rehabilitation, 41: 71-75, 1960.

Bowers D and Heilman KM. Pseudoneglect: effects of hemispace on a tactile line-bisection task. Neuropsychologia, 18: 491-498, 1980.

Bridgeman B, Peery S, and Anand S. Interaction of cognitive and sensorimotor maps of visual space. Perception and Psychophysics, 59: 456-469, 1997.

Bridgeman B, Gemmer A, Forsman T, and Huemer V. Processing spatial information in the sensorimotor branch of the visual system. Vision Research, 40: 3539-3552, 2000.

Bruno N. When does action resist visual illusions? Trends in Cognitive Sciences, 5: 379-382, 2001.

Carey DP. Do action systems resist visual illusions? Trends in Cognitive Sciences, 5: 109-113, 2001.

Edwards MG and Humphreys GW. Pointing and grasping in unilateral visual neglect: effect of on-line visual feedback in grasping. Neuropsychologia, 37: 959-973, 1999.

Ellis RR, Flanagan JR, and Lederman SJ. The influence of visual illusions on grasp position. Experimental Brain Research, 125: 109-114, 1999.

Franz VH, Gegenfurtner KR, Bulthoff $\mathrm{HH}$, and Fahle M. Grasping visual illusions: no evidence for a dissociation between perception and action. Psychological Science, 11: 20-25, 2000.

Haffenden AM and Goodale MA. The effect of pictorial illusion on prehension and perception. Journal of Cognitive Neuroscience, 10: 122-136, 1998.

Heilman $\mathrm{KM}$ and Van den Abel T. Right hemispheric dominance for attention: the mechanism underlying hemispheric asymmetries of inattention (neglect). Neurology, 30: 327-330, 1980.

Hjaltason H, Caneman G, and Tegner R. Visual and tactile rodbisection in unilateral neglect. Cortex, 29: 583-588, 1993.

$\mathrm{Hu}$ Y and Goodale MA. Grasping after a delay shifts size-scaling from absolute to relative metrics. Journal of Cognitive Neuroscience, 12: 856-868, 2000.

Hughes LE. Visual Perception, Attention and Action. Unpublished PhD, Sydney: Macquarie University, 2005.

Hughes LE, Bates TC, and Aimola Davies A. Grasping at sticks: dissociations between perception and action. Experimental Brain Research, 157: 397-402, 2004.

Hughes LE, Bates TC, and Aimola Davies AM. The effects of local and global processing demands on perception and action. Brain and Cognition, 59: 71-81, 2005.

Jackson SR, Jones CA, Newport R, and Pritchard CA. Kinematic analysis of goal-directed prehension movements executed under binocular, monocular, and memory-guided viewing conditions. Visual Cognition, 4: 113-142, 1997.

Jackson SR, Newport R, and Shaw A. Monocular vision leads to a dissociation between grip force and grip aperture scaling during reach-to-grasp movements. Current Biology, 12: 237240, 2002.
Jeannerod M and Biguer B. The Directional Coding of Reaching Movements. A Visuomotor Conception of Spatial Neglect. North Holland: Elsevier Science Publishers, 1987.

Jewell G and McCourt ME. Pseudoneglect: a review and metaanalysis of performance factors in line-bisection tasks. Neuropsychologia, 38: 93-110, 2000.

Kinsbourne $\mathrm{M}$. The cerebral basis of lateral asymmetries in attention. Acta Psychologica, 33: 193-201, 1970.

Loftus A, Servos P, Goodale MA, Mendarozqueta N, and MonWilliams M. When two eyes are better than one in prehension: monocular viewing and end-point variance. Experimental Brain Research, 158: 317-327, 2004.

Marotta JJ, Behrmann M, and Goodale MA. The removal of binocular cues disrupts the calibration of grasping in patients with visual form agnosia. Experimental Brain Research, 116: 113-121, 1997.

Marotta JJ, DeSouza JF, Haffenden AM, and Goodale MA. Does a monocularly presented size-contrast illusion influence grip aperture? Neuropsychologia, 36: 491-497, 1998.

Mesulam MM. Spatial attention and neglect: parietal, frontal and cingulate contributions to the mental representation and attentional targeting of salient extrapersonal events. Philosophical Transactions of the Royal Society of London. Series B, Biological Sciences, 354: 1325-1346, 1999.

Milner AD and Goodale MA. The Visual Brain in Action. Oxford: Oxford University Press, 1995.

McIntosh RD, McClements KI, Dijkerman HC, Birchall D, and Milner AD. Preserved obstacle avoidance during reaching in patients with left visual neglect. Neuropsychologia, 42: 1107-1117, 2004.

Mon-Williams M and Dijkerman HC. The use of vergence information in the programming of prehension. Experimental Brain Research, 128: 578-582, 1999.

Mon-Williams M, Tresilian JR, McIntosh RD, and Milner AD. Monocular and binocular distance cues: insights from visual form agnosia I (of III). Experimental Brain Research, 139: 127-136, 2001.

Oldfield RC. The assessment and analysis of handedness: the Edinburgh inventory. Neuropsychologia, 9: 97-114, 1971.

Reuter-Lorenz PA, Kinsbourne M, and Moscovitch M. Hemispheric control of spatial attention. Brain and Cognition, 12: 240-266, 1990.

Robertson IH, Nico D, and Hood BM. The intention to act improves unilateral left neglect: two demonstrations. Neuroreport, 7: 246-248, 1995.

Robertson IH. Cognitive rehabilitation: attention and neglect. Trends in Cognitive Sciences, 3: 385-393, 1999.

Rossetti Y, Revol P, McIntosh R, Pisella L, Rode G, Danckert J, Tilikete C, Dijkerman HC, Boisson D, Vighetto A, Michel F, and Milner AD. Visually guided reaching: bilateral posterior parietal lesions cause a switch from fast visuomotor to slow cognitive control. Neuropsychologia, 43: 162-177, 2005.

Schindler I, Rice NJ, McIntosh RD, Rossetti Y, Vighetto A, and Milner AD. Automatic avoidance of obstacles is a dorsal stream function: evidence from optic ataxia. Nature Neuroscience, 7: 779-784, 2004.

Servos P and Goodale MA. Binocular vision and the on-line control of human prehension. Experimental Brain Research, 98: 119-127, 1994.

Servos P, Goodale MA, and Jakobson LS. The role of binocular vision in prehension: a kinematic analysis. Vision Research, 32: 1513-1521, 1992.

Smeets JBJ, Brenner E, de Grave DDJ, and Cuijpers RH. Illusions in action: consequences of inconsistent processing of spatial attributes. Experimental Brain Research, 147: 135-144, 2002. 
Westwood DA, Chapman CD, and Roy EA. Pantomimed actions may be controlled by the ventral visual stream. Experimental Brain Research, 130: 545-548, 2000b.

Westwood DA and Goodale MA. Perceptual illusion and the real-time control of action. Spatial Vision, 16: 243-254, 2003.
Westwood DA, Heath M, and Roy EA. The effect of a pictorial illusion on closed-loop and open-loop prehension. Experimental Brain Research, 134: 456-463, 2000a.

Westwood DA, McEachern T, and Roy EA. Delayed grasping of a Muller-Lyer figure. Experimental Brain Research, 141: 166-173, 2001. 